\title{
Fast Optical Control of Spin in Semiconductor Interfacial Structures
}

\author{
L. Nádvorník, ${ }^{1,2, *}$ M. Surýnek, ${ }^{3}$ K. Olejník, ${ }^{1}$ V. Novák, ${ }^{1}$ J. Wunderlich, ${ }^{1,4}$ F. Trojánek, ${ }^{3}$ T. Jungwirth,,${ }^{1,5}$ and P. Němec ${ }^{3}$ \\ ${ }^{1}$ Institute of Physics ASCR, v.v.i., Cukrovarnická 10, 16253 Praha 6, Czech Republic \\ ${ }^{2}$ Department of Physical Chemistry, Fritz. Haber Institute of the Max Planck Society, \\ 14195 Berlin, Germany \\ ${ }^{3}$ Faculty of Mathematics and Physics, Charles University, Ke Karlovu 3, 12116 Praha 2, Czech Republic \\ ${ }^{4}$ Hitachi Cambridge Laboratory, J. J. Thomson Avenue, CB3 OHE Cambridge, United Kingdom \\ ${ }^{5}$ School of Physics and Astronomy, University of Nottingham, Nottingham NG7 2RD, United Kingdom \\ (Received 1 June 2017; revised manuscript received 9 August 2017; published 25 September 2017)
}

We report on a picosecond-fast optical removal of spin polarization from a self-confined photocarrier system at an undoped $\mathrm{GaAs} /(\mathrm{Al}, \mathrm{Ga}) \mathrm{As}$ interface possessing superior long-range and high-speed spintransport properties. We employ a modified resonant-spin-amplification technique with unequal intensities of subsequent pump pulses to experimentally distinguish the evolution of spin populations originating from different excitation laser pulses. We demonstrate that the density of spins, which is injected into the system by means of the optical orientation, can be controlled by reducing the electrostatic confinement of the system using an additional generation of photocarriers. It is also shown that the disturbed confinement recovers within hundreds of picoseconds after which spins can be again photoinjected into the system.

DOI: $10.1103 /$ PhysRevApplied.8.034022

\section{INTRODUCTION}

Magnetic random access memory (MRAM) bits [1] and spin Hall effect transistors [2] are examples of two different conceptual approaches to spintronic devices whose functionality requires different spin-conserving length scales. The operation of MRAM bits is based on a vertical transfer of spins through a nanometer-thin layer in a "sandwichlike" layered structure [3]. In spin-logic devices, however, spins have to be manipulated (by electric gates, for instance) before they reach the drain electrode, which calls for a lateral design rather than the vertical geometry [4-6]. An orders-of-magnitude-larger spinconserving length scale is, therefore, needed in the spinlogic devices due to the typical dimensions of the order of a micrometer of lateral structures fabricated by electronbeam lithography [2,7-9]. The desired high-rate operation of the devices implies an additional requirement on a fast spin transport over this length scale. The criteria for the simultaneously long-range and high-speed spin transport are not satisfied in a majority of standard systems, including $n$-doped bulk semiconductors [10-12] or (001)-grown quantum wells [13], due to their limited spin mobility or spin lifetime, respectively. Another critical parameter which limits the operation rate of a spin-logic device is the speed of spin manipulation. While the spin injection and detection can be fast processes (for instance, by means of the optical orientation [14] and magneto-optical probing $[13,15])$, a fast removal of the spin polarization from the system is a much more challenging task.

"nadvl@fzu.cz
In this paper, we show experimentally that the electronic spin polarization can be removed from a transport channel of a self-confined system in a picosecond time scale using a control optical pulse which reduces the level of its confinement. We demonstrate this functionality in an optically generated self-confined system formed at an undoped $\mathrm{GaAs} / \mathrm{Al}_{x} \mathrm{Ga}_{1-x}$ As heterointerface where the spin transport meets the other key requirements of high speed and long range, as reported recently in Ref. [16]. In addition, we show that the confinement of the transport layer is recovered after the removal of the spin polarization within a few hundreds of picoseconds. Combining all these favorable characteristics, we obtain a unique candidate system for the development of the spin-logic concept in semiconductors.

Our study is performed by a modified resonant-spinamplification (RSA) technique [10] using unequal intensities of neighboring pump pulses which enables us to probe simultaneously the time evolution of mixed spin populations created at different time instants. This scheme also allows us to generate the spin polarization by a lowerintensity pulse in a well-controlled way without disturbing the confinement, then to reduce it by a high-intensity pulse, and eventually to read it by a probe pulse.

The paper is organized as follows: First, we describe the studied structure and explain the mechanism of the creation of the long-lived electronic spin subsystem and show its fingerprint in a magneto-optical signal. Then we describe the modified RSA technique with unequal intensities of subsequent pump pulses. In the following sections, we discuss our experimental results demonstrating the fast optical control of spin polarization. Finally, in Appendixes, 
we provide additional information on our experimental setup and an estimate of the impact of the disturbed confinement on the spin polarization.

\section{STUDIED SYSTEM}

\section{A. Sample composition}

The studied long-lived and highly mobile electron spin system is self-confined near the upper $\mathrm{GaAs} / \mathrm{Al}_{x} \mathrm{Ga}_{1-x} \mathrm{As}$ interface in an undoped heterostructure [16]. This spin system is formed in a wide range of $x$ composition and layer thicknesses as a consequence of the spatial separation of optically generated electron-hole pairs in a built-in electric field due to surface states. In this paper, we use the optimal $\mathrm{Al}$ composition, $x=0.4$, and the most simple layer composition shown in Fig. 1(a). A 100-nm-thick undoped $\mathrm{Al}_{0.4} \mathrm{Ga}_{0.6} \mathrm{As}$ barrier is deposited by molecularbeam epitaxy on top of an insulating GaAs buffer and a $\mathrm{GaAs}$ substrate. The barrier is then covered by another undoped 800-nm-thick GaAs layer where the confined electron subsystem is formed, as explained below.

\section{B. Creation of electronic subsystem}

The process which usually limits the spin lifetime $\tau_{s}$ in undoped structures is the electron-hole recombination [10]. The origin of the unusually long $\tau_{s}$ measured in our undoped sample is the suppression of the recombination spin-decay channel due to the presence of a long-lived subsystem of photogenerated excess electrons. This effective optically generated doping is possible due to the spatial separation of photogenerated electrons and holes in the built-in electric field. If we consider our structure in dark, charged residual bulk and surface states [17] pin the Fermi level near the valence band [16], which causes a band bending and, thus, an electric field across the structuresee Fig. 1(b), solid curves. When the sample is illuminated from the surface side, photoelectrons and photoholes are generated. If the structure did not contain the barrier, the photocarriers would migrate in the built-in electric field, fill all the charged states, and compensate fully the electric field. However, because of the (Al,Ga)As barrier, the free migration between the layers above and below the barrier is obstructed, and the full compensation of the field is not possible (dotted curves). The residual built-in electric field leads to the formation of a steady-state optically excited electronic system near the upper $\mathrm{GaAs} /(\mathrm{Al}, \mathrm{Ga}) \mathrm{As}$ interface, depicted by the quasi-Fermi level in Fig. 1(b). Because of the minimal spatial overlap between electrons and holes, the carrier recombination is suppressed. The long-lived system created this way can be viewed as an effective (optically generated) local $n$ doping which suppresses the spin-decay channel via the carrier recombination and allows for the observed long $\tau_{s}$, similarly as in $n$-doped GaAs samples [10,11]. The process is described explicitly in Sec. V; a more detailed discussion with its
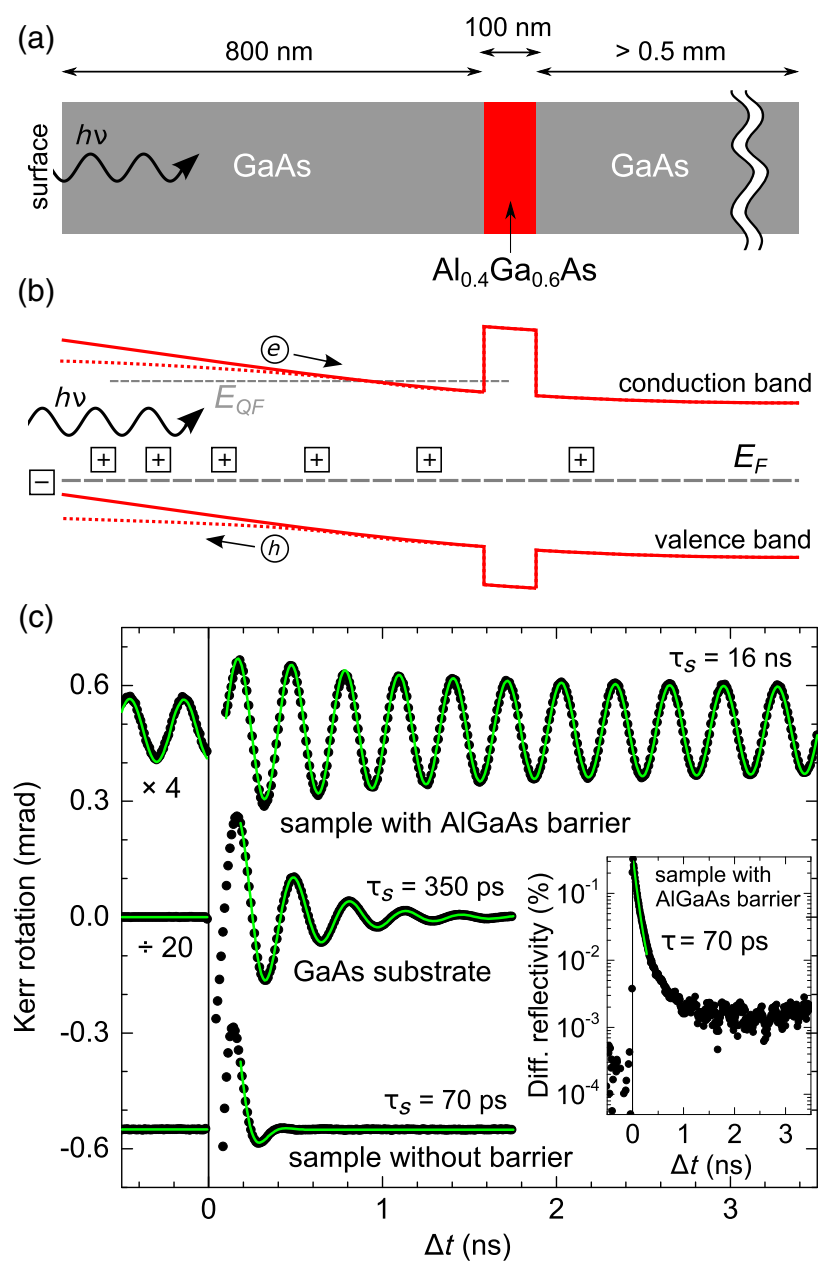

FIG. 1. (a) The layer structure of the studied sample (the sample surface, on which the light is incident, is on the left side). (b) The energetic diagram of the conduction and valence bands before (solid curves) and after the illumination (dotted curves). The Fermi level $E_{F}$ is pinned to the edge of the valence band due to the negatively charged surface states (the square with the minus sign) and ionized unintentional bulk impurity states (squares with the plus sign), and the band bending results in a built-in electric field. After the illumination by light with photon energy $h \nu=1.52 \mathrm{eV}$, the photocreated electrons (circle with the letter $e$ ) and holes (circle with $h$ ) are driven by the built-in electric field and spatially separated (arrows). This process compensates partially the electric field and leads to the formation of a steady-state long-lived electron subsystem near the upper $\mathrm{GaAs} /(\mathrm{Al}, \mathrm{Ga}) \mathrm{As}$ interface, depicted by the quasi-Fermi level $E_{Q F}$. The electron-hole overlap is minimal in the subsystem which suppresses the recombination and allows for long spin lifetime $\tau_{s}$. (c) The measured dynamics of the magneto-optical Kerr signal (points) with fits by Eq. (1) (solid curves). The data measured in the corresponding samples at $10 \mathrm{~K}, B=500 \mathrm{mT}, h \nu=1.52 \mathrm{eV}$, and $14 \mu \mathrm{J} \mathrm{cm}^{-2}$ are multiplied or divided by the indicated factors and vertically shifted for clarity. The nonzero signal for $\Delta t<0$ in the sample with the (Al,Ga)As barrier is a signature that $\tau_{s}$ in the electron subsystem exceeds the time spacing between the neighboring pumplaser pulses $t_{0}$, which is $12.5 \mathrm{~ns}$ in this particular case. Inset: The measured dynamics of the differential reflectivity (points) for the sample with the (Al,Ga)As barrier. The depicted recombination time $\tau$ is inferred from an exponential fit (curve). 
experimental confirmation can be found in Ref. [16] and its Supplemental Material.

\section{Time-resolved magneto-optical detection}

The presence of the steady-state long-lived electronic spin system in the studied sample is revealed by the magneto-optical (MO) pump-and-probe experiment; we use the optical orientation and the MO Kerr effect for spin injection and detection, respectively, as described in detail in Appendix A and Fig. 5. In Fig. 1(c), we show the data measured in the sample with the confined electronic subsystem and in two reference samples using laser pulses with a repetition frequency of $80 \mathrm{MHz}$, i.e., a time spacing between neighboring laser pulses $t_{0}=12.5 \mathrm{~ns}$. Clearly, the presence of the $(\mathrm{Al}, \mathrm{Ga}) \mathrm{As}$ barrier is essential for the achievement of the long spin lifetime. In fact, even without any quantitative analysis, the presence of the MO signal at negative pump-probe time delays $\Delta t$ (i.e., the fact that the spin signal induced by a previous pump pulse does not decay to zero before the impact of an another pump pulse) immediately indicates that $\tau_{s} \gtrsim t_{0}=12.5 \mathrm{~ns}$. For comparison, the typical recombination time $\tau$ is of the order of hundreds of picoseconds in the undoped GaAs.

The fitting of the data provides a more precise determination of $\tau_{s}$ as shown in Fig. 1(c). By applying the model for the total MO signal presented in Ref. [16] [i.e., Eq. (1) for $m=0,1$ and $A_{0}=A_{1}=A$ below] on the data [solid curves in Fig. 1(c)], we deduced $\tau_{s} \approx 16 \mathrm{~ns}$, which is, indeed, more than 2 orders of magnitude larger than the carrier recombination time $\tau \approx 70 \mathrm{ps}$, inferred from the dynamics of the differential reflectivity measured in this sample (inset). The nonzero reflectivity signal at times after this bulk-carrier recombination indicates the presence of the long-lived subsystem. From the dependence of the fitted oscillatory (Larmor) frequency on the applied magnetic field, we infer the $g$ factor of magnitude $0.45 \pm 0.01$, confirming that the spin carriers are free electrons $[18,19]$. In the bare GaAs substrate and the undoped epitaxial GaAs on the substrate, the spin lifetimes are more than 2 orders of magnitudes shorter-350 and $70 \mathrm{ps}$ for the substrate and epitaxial GaAs, respectively. This agrees well with the observed $\tau$ in the sample with the barrier and with typical values reported for undoped bulk GaAs, where the spin lifetime of spin-polarized photocarriers is limited by their recombination time and where $\tau$ is typically reported at the time scale of hundreds of picoseconds $[10,13,20]$. The inferred $g$ factors are the same within the experimental error for all three samples. This confirms that the confining potential in the studied sample is relatively weak unlike in triangular quantum wells, for example, where a shift of $g$ from the bulk value is observed [21].

We point out that the MO detection technique enables us to separate signals coming from different parts of the studied sample. In particular, a proper selection of the probe detection wavelength enhances the sensitivity to the detected MO signals originating from the confined electronic subsystem (see Appendix A, Ref. [16], and its Supplemental Material for more details). Thanks to this, the measured MO dynamics is not dominated by contributions of short-lived spins generated in the bulk despite their much higher density [see Fig. 1(c)]. On the other hand, the differential reflectivity does not have such selectivity, and the signal reveals the dynamics of the overall carrier density, which is dominated by the bulk-related contributions.

\section{RSA TECHNIQUE WITH UNEQUAL INTENSITIES OF SUBSEQUENT LASER PULSES}

In our case, when the electron spin lifetime is longer than the time separation between adjacent pump-laser pulses, the measured MO signal is a sum of signals coming from spin populations photoinjected by pump-laser pulses at different times. The separation of these signals, which is difficult to infer from a time-domain measurement, can be performed straightforwardly by the RSA technique [10] that is schematically depicted in Fig. 2(a). The total Kerr signal $S(\Delta t, B)$ can be expressed as

$$
\begin{aligned}
S(\Delta t, B)= & \sum_{m} \Theta\left(\Delta t+m t_{0}\right) A_{m} e^{-\left(\Delta t+m t_{0}\right) / \tau_{s}} \\
& \times \cos \left[g \mu_{B} B\left(\Delta t+m t_{0}\right) / \hbar\right],
\end{aligned}
$$

where $\Theta(x)$ is the Heaviside function that guarantees that only the previous pulses contribute to the total signal and $m=0,1,2, \ldots$ is the index of the $m$ th preceding excitation pulse. $A_{m}$ is the initial amplitude of the Kerr signal at the instant of creation of the respective spin population by the $m$ th pulse (for equal intensities of all excitation laser pulses, $A_{m}=A$ is the same for all $m$ ). The term $g \mu_{B} B / \hbar$ is the Larmor frequency due to the external magnetic field $B$ applied in the sample plane where $g, \mu_{B}$, and $\hbar$ are the $g$ factor corresponding to the studied system, Bohr magneton, and reduced Planck constant, respectively.

As follows from Eq. (1), if $\Delta t$ is fixed and $B$ is varied instead, two spin populations created by two subsequent excitation pulses contribute to the total Kerr signal with different oscillatory frequencies. This is due to a different time elapsed from the instant of the photoinjection of the corresponding spin populations [see Fig. 2(a)]. Consequently, the signal amplitudes corresponding to the different modulation frequencies are connected with the number of spins in these two populations. Therefore, one can probe simultaneously the time evolution of many mixed-spin populations created at different instants by measuring the RSA curves at several $\Delta t$.

The standard implementation of the RSA technique is based on utilization of a train of laser pulses with equal intensities [10]. Here, on the contrary, we use a train of laser pulses where each second laser pulse has a significantly lower intensity using a nonideally working pulse picker-see 


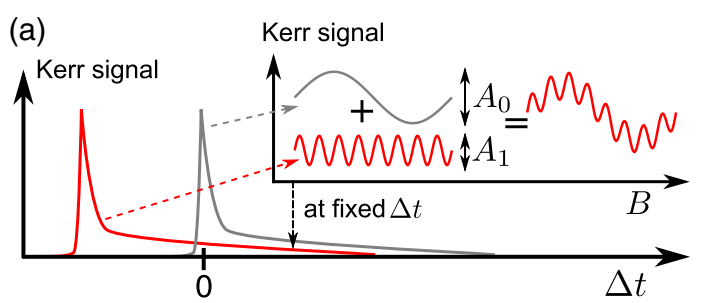

(b)

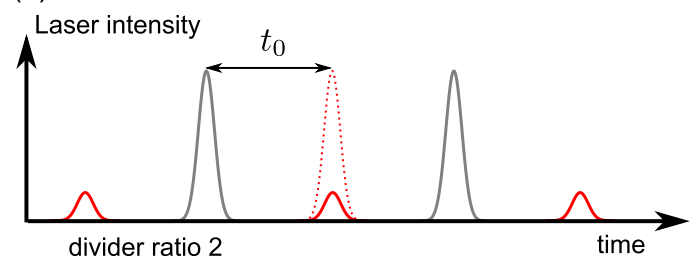

FIG. 2. (a) A schematic depiction of the RSA technique. If the decay of the spin population exceeds the time spacing between neighboring laser pulses, the MO signal measured for a given value of a time delay $\Delta t$ is a sum of (at least) two signals. Inset: Dependence of the MO signal for a fixed value of $\Delta t$ on external magnetic field $B$. (b) A schematic illustration of the train of femtosecond laser pulses where each second laser pulse has a significantly lower intensity (in our case at least 30 times) due to the nonideally working pulse picker which does not remove completely the laser pulse depicted by a dotted line (see Appendix A for more details).

Fig. 2(b) and Appendix A. The lower-intensity laser pulses create a confined spin population at the $\mathrm{GaAs} /(\mathrm{Al}, \mathrm{Ga}) \mathrm{As}$ interface without disturbing the self-confinement, as discussed in Sec. II B. A spin population created in such a way is already saturated with respect to the laser fluence, as can be seen in the inset in Fig. 4(a). This spin population serves as a probe of the influence of the much stronger (unattenuated) laser pulse which is incident on the sample at $\Delta t=0 \mathrm{ps}$. We note that this modified RSA technique with unequal intensities of neighboring laser pulses is, in a certain sense, using the same idea as an ordinary pump-probe experiment where a weak probe pulse is used to measure the changes induced in the sample by a strong pump pulse.

\section{RESULTS}

In Fig. 3, we show the results of our modified RSA technique for several values of time delays measured on the studied sample (see Sec. II A). For $\Delta t=500$ and $150 \mathrm{ps,}$ we can identify two signals with distinct frequencies - the larger signal oscillating with a smaller frequency corresponds to the spin population photoinjected by an unattenuated pulse at $\Delta t=0$, and the smaller signal oscillating with a larger frequency corresponds to the spin population photoinjected by a reduced-intensity pulse at $\Delta t=-t_{0}=$ $-12.5 \mathrm{~ns}$. On the other hand, for $\Delta t=-400 \mathrm{ps}$ [Figs. 3(e) and $3(\mathrm{f})]$ we see only one precession frequency, because only the spin population photoinjected by the reducedintensity pulse is present in the sample for this time delay,

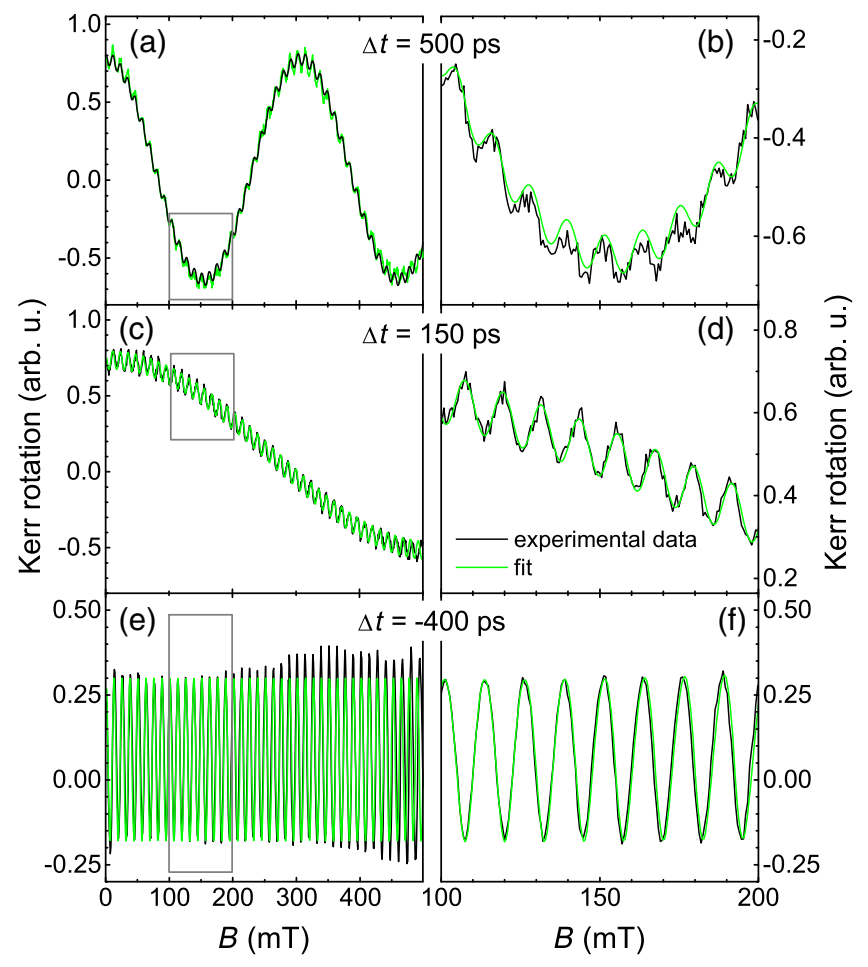

FIG. 3. Results obtained by the RSA technique using a train of laser pulses where each second laser pulse has a significantly lower intensity. The experimentally measured data (black lines) are measured at $\Delta t=500$ (a),(b), 150 (c),(d), and $-400 \mathrm{ps}(\mathrm{e}),(\mathrm{f})$; the right column shows enlarged views of the data marked by gray rectangles in the left column. The fits of the data by Eq. (1) for $m=0$ and 1 are plotted by the green (gray) curves. All data are measured at $10 \mathrm{~K}$ using laser fluence $140 \mu \mathrm{J} \mathrm{cm}^{-2}$ and a time separation of unattenuated pulses of $2 t_{0}=25 \mathrm{~ns}$.

which is in agreement with the deduced value of the spin lifetime $\tau_{s} \sim 16 \mathrm{~ns}<2 t_{0}=25 \mathrm{~ns}$. Moreover, a strong reduction of the spin population photoinjected by the reduced-intensity pulse due to the impact of the unattenuated pulse is immediately apparent from a comparison of the oscillation amplitudes in Figs. 3(f) and 3(d). To visualize this effect more clearly, we fit the measured data (see Fig. 3) by Eq. (1) for $m=0$ and 1.

If there are no disturbing effects of the incident laser pulses, the time-delay-dependent amplitudes $A_{m}(\Delta t)$ should follow the exponential decay due to the electron spin relaxation

$$
A_{m}(\Delta t)=A_{m} e^{-\left(\Delta t+m t_{0}\right) / \tau_{s}}
$$

for $m=0$ and 1 according to Eq. (1). However, as illustrated in Fig. 4(b), $A_{1}(\Delta t)$ does not follow the dependence expected from Eq. (2), which is shown by the red dotted curve in Fig. 4(b). Instead, the impact of the unattenuated pulse reduces it almost instantly, within $\Delta t<6 \mathrm{ps}$, to one-half of its value. Following this, the decrease of $A_{1}$ then slows down, and it saturates at 


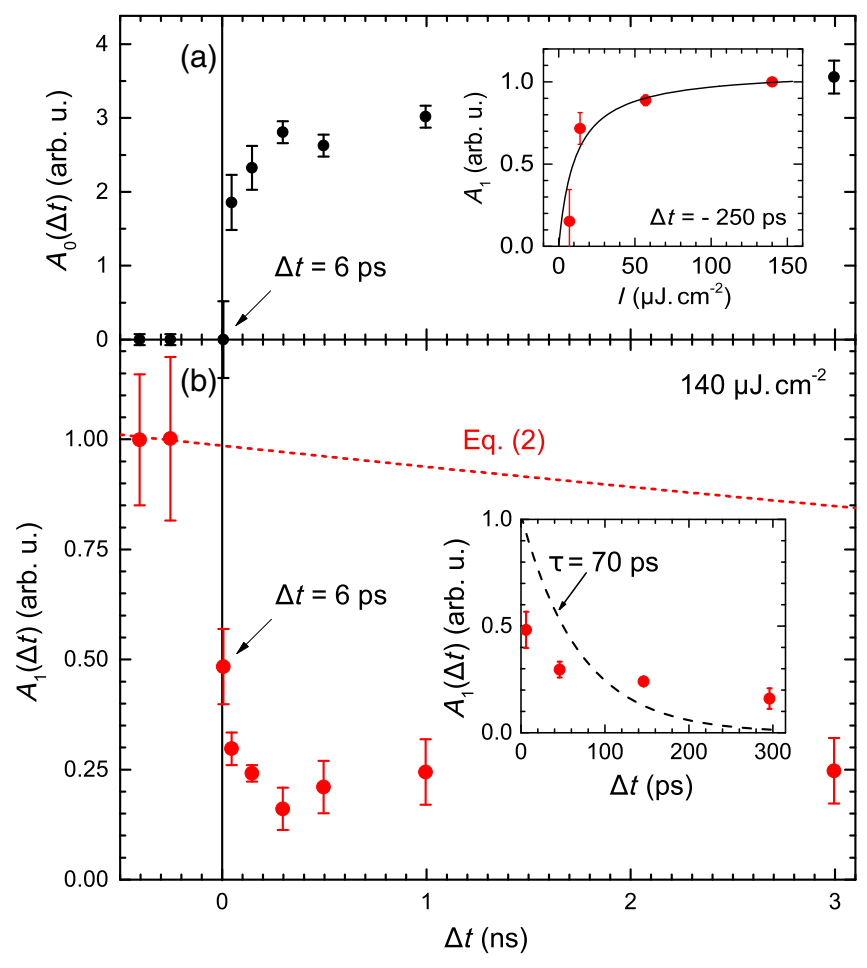

FIG. 4. Time evolution of spin populations photoinjected by laser pulses. (a) Amplitude $A_{0}(\Delta t)$ generated by the unattenuated pulse at $\Delta t=0 \mathrm{ps}$. (b) Amplitude $A_{1}(\Delta t)$ generated by the diminished pulse at $\Delta t=-12.5 \mathrm{~ns}$. The red dotted curve depicts the expected exponential spin decay if no disturbing effects of the incident laser pulses is assumed [Eq. (2)]. Insets: (a) The dependence of $A_{1}$ on the fluence measured at $\Delta t=-250 \mathrm{ps}$. Because of a very weak (and not known precisely) intensity of the suppressed laser pulses, the $x$ scale is expressed in terms of the intensity of the unattenuated laser pulses, which is used to label the excitation conditions throughout the paper. The curve is a fit by a model based on the rate equation for an energy level with a limited number of states (see Ref. [22] for details). (b) An enlargement of data for small time delays with a calculated decay of the spin population which would correspond to the electronhole recombination with $\tau \approx 70$ ps (black dashed curve). The experiment is performed at $10 \mathrm{~K}$ using laser fluence $140 \mu \mathrm{J} \mathrm{cm}^{-2}$ and a time separation of unattenuated pulses of $2 t_{0}=25 \mathrm{~ns}$.

approximately $1 / 4$ of the original value within approximately $1 \mathrm{~ns}$. Naturally, the amplitude $A_{0}(\Delta t)$ is zero for $\Delta t<0$, but, more interestingly, no spin population is injected to the subsystem within the first 6 ps after the laser-pulse impact. In fact, it takes tens of picoseconds before the spin population starts to increase [Fig. 4(a)], and it saturates slowly on a nanosecond time scale.

We completed our time-resolved experiments with a time-averaged measurement of the nuclear spin polarization due to the presence of spin-polarized photocarriers. The experiment is described in detail in Appendix B. By measuring the change of the Larmor frequency of electronic spins, we determine the effective magnetic field $B_{n}$ generated by spin-polarized nuclei which adds to the external magnetic field. Using this steady-state $B_{n}$, we roughly estimate the time-averaged degree of spin polarization of electrons $P_{e}=\left(n_{+}-n_{-}\right) /\left(n_{+}+n_{-}\right)$, which is given by the average density $n_{+}$or $n_{-}$of electrons with their spin oriented parallel or antiparallel to the propagation vector of excitation light, respectively. We infer $P_{e} \approx 18 \%$ and a nuclei charging time of approximately $60 \mathrm{~min}$.

\section{DISCUSSION}

In the previous section, we show experimental data demonstrating that a strong excitation pulse triggers a very fast reduction of the existing spin population $A_{1}$. In the following text, we discuss possible mechanisms that can be responsible for this observation.

First, we describe the processes which take place if a train of circularly polarized laser pulses is absorbed in an $n$-type doped bulk semiconductor. Because of the optical orientation, each laser pulse excites a nonequilibrium population of spin-polarized electrons and holes which recombine with a time constant $\tau$. Thanks to the presence of equilibrium electrons in the conduction band, which are provided by the doping, a certain fraction of the photoinjected holes recombines with these preexisting electrons. Consequently, the remaining photoinjected spin-polarized electrons can maintain their spin for time $\tau_{s}$ significantly exceeding $\tau[10,11]$. For $\tau_{s}$ larger than the spacing between the adjacent laser pulses $t_{0}$, the resulting electronic spin population is always partially reduced by an impact of the following laser pulse due to a recombination with photoinjected holes, and new spin-polarized electrons are added, which is leading to a periodic spin renewal.

In our sample, the observed fast reduction of the spin population $A_{1}$ [to approximately $50 \%$ of the initial value in time $<6$ ps as shown in the inset in Fig. 4(b)] cannot be explained by the recombination of the existing electrons with the photoinjected holes. This is because we observed experimentally that the fastest recombination time in our system is $\tau=70 \mathrm{ps}$ [see the inset in Fig. 1(c) for the transient reflectivity decay measurement], a value consistent with the literature $[10,13,20]$. Moreover, a recombination of electrons would free some states in the studied self-confined subsystem, which are nearly fully occupied by electrons for our experimental conditions [see the saturation behavior of the MO signal with the pump intensity in the inset in Fig. 4(a) [22]]. Consequently, the fast reduction of $A_{1}$ due to a recombination of electrons should be accompanied by an increase of newly injected population $A_{0}$ into the subsystem, which is not the case [see Fig. 4(a), where $A_{0} \approx 0$ for $\Delta t=6 \mathrm{ps}$ ].

Another possible mechanism, which could in principle reduce $A_{1}$ without a decay of the electron concentration, is the spin-relaxation process. The most efficient mechanism in GaAs at low temperatures, especially in confined systems, is the Dyakonov-Perel (DP) spin dephasing [23]. Indeed, the DP mechanism can be the origin of a 
fast spin relaxation with $\tau_{s}$ of tens or even units of picoseconds in systems with a strong confinement such as remote-doped $(\mathrm{Al}, \mathrm{Ga}) \mathrm{As} / \mathrm{GaAs}$ heterointerfaces or quantum wells (see, e.g., Ref. [13] and references therein). However, we observe exceptionally long $\tau_{s} \approx 16 \mathrm{~ns}$, which is a signature of a weak confinement and an inefficient DP dephasing in the studied sample [16]. The photoexcitation of carriers due to the absorption of an intense excitation pulse can be expected to weaken the confinement [see Fig. 1(b)]. Moreover, the eventual pump-induced fluctuations of the confinement would lead to a further decrease of the DP efficiency due to motional narrowing $[23,24]$, and, thus, it would lead to a further increase of $\tau_{s}$. Overall, this rules out the DP and other less-efficient spin-relaxation mechanisms as a cause of the fast decay of $A_{1}$.

We attribute the observed phenomenon to a removal of the spin-polarized electrons from the self-confined electron subsystem. This is due to the pump-induced disturbance of the confining potential and, thus, to a reduction of the density of available states in the subsystem. The confinement is a self-consistent process which depends strongly on boundary conditions and local charge distribution and can be disturbed by a high density of photoinjected charges [16]. When the confining potential is modified by the additional carriers in the bulk GaAs, the excess electrons move from the subsystem to the bulk [see Fig. 1(b)]. Therefore, they do not contribute significantly to the measured MO signals, which were optimized for the subsystem detection as discussed in Sec. II C and in more detail in Appendix A. In the following tens and hundreds of picoseconds, the electrostatic confinement starts to recover due to a continuous decay of photocarriers in the bulk via their recombination, and, consequently, additional states become available in the subsystem. These new states and the states made available by a partial recombination of $A_{1}$ electrons with the photoinjected holes are subsequently filled by spin-polarized electrons from the bulk. This results in an increase of the spin-polarized population $A_{0}$ within approximately 300 ps (see Fig. 4). To sum up, this experiment reveals that the spin polarization can be periodically "erased" from the superior highly mobile spin-transport layer in times $<6 \mathrm{ps}$ and regenerated again in hundreds of picoseconds. This defines the constraints for the high-rate operation of spin-logic devices based on this structure.

A side effect of this functionality is an unideal renewal of the spin system due to the incomplete decay of $A_{1}$ and other possible perturbations of the spin system. This leads to a decrease of the degree of spin polarization $P_{e}$ which is practically achievable in the subsystem. Ideally, each absorbed laser pulse should generate $P_{e} \approx 50 \%$ at the instant of absorption [25]. If a material with $\tau_{s} \approx 16 \mathrm{~ns}$ is excited by a train of circularly polarized laser pulses with a repetition rate of $80 \mathrm{MHz}$, it should lead to time average
$P_{e} \approx 36 \%$ when this unideal renewal is not considered. Experimentally, we obtain a rough estimate of $P_{e} \approx 18 \%$ that shows, indeed, a visible but not dramatic reduction of the spin polarization.

Based on the properties of our system demonstrated in this and a previous study [16], we believe that the undoped $\mathrm{GaAs} /(\mathrm{Al}, \mathrm{Ga}) \mathrm{As}$ interface represents a unique kind of structure from the spintronic point of view. The closest systems with comparable or even slightly better spintransport properties are (110)-grown GaAs quantum wells $[26,27]$, where spin-transport distances can reach $100 \mu \mathrm{m}$ with an electron mobility of $10^{5}-10^{6} \mathrm{~cm}^{2} \mathrm{~V}^{-1} \mathrm{~s}^{-1}$. However, in these systems, the confinement of carriers is strong and robustly defined by the doping, and, thus, the spin polarization cannot be removed from the quantum well using the same fast mechanism as in our case. In principle, an alternative way of generation and control of the confinement is the electrical gating of an undoped semiconductor interface, as reported in Ref. [28] on a spin system in a $\mathrm{GaAs} /(\mathrm{Al}, \mathrm{Ga}) \mathrm{As}$ structure. However, here, the speed of the confinement modulation is limited by the bandwidth of the current electronics, which is much slower than using optical methods. On the other hand, the philosophy of our approach should be applicable on any similar semiconductor interface and should be possible to optimize it to even better performance.

\section{CONCLUSION}

Long-range and high-speed spin transport together with fast spin manipulation are the keystones of high-operationrate spin-logic devices. In this paper, we show that the fast spin removal functionality can be added to a superior spintransport channel in undoped $\mathrm{GaAs} / \mathrm{Al}_{x} \mathrm{Ga}_{1-x}$ As heterointerfaces using absorption of optical pulses. By employing a modified resonant-spin-amplification technique with unequal intensities of neighboring laser pulses, we demonstrate that spins can be removed from the high-performance self-confined system in a picosecond time scale by optical manipulation of the densities of carriers in the system. This is achieved by reducing the degree of confinement due to the photoinjection of carriers in this region. It is also observed that the recovery of the confinement occurs in a few hundreds of picoseconds. The demonstrated functionality which allows for a fast periodic erasing and regenerating of the electronic spin polarization in a long-range and high-speed spin-transport layer makes the system an excellent candidate for fast spin-logic devices. Since these superior properties should be, in principle, common for a larger variety of selfconfined semiconductor structures, the present finding can open perspectives for spin-logic applications.

\section{ACKNOWLEDGMENTS}

We acknowledge support from the European ERC Synergy Grant No. 610115, the Ministry of Education of 
the Czech Republic Grants No. LM2015087 and LNSMLNSpin, from the Czech Science Foundation Grant No. 14$37427 \mathrm{G}$, and from the Charles University Grants No. 1582417 and No. SVV-2017-260445.

\section{APPENDIX A: EXPERIMENTAL SETUP}

The used time-resolved spin-injection and detection method relies on the pump-probe technique [29]. The spin injection is realized using optical orientation [14] by the circularly polarized excitation laser pulse, and the spin detection is done by measuring the polarization rotation of the linearly polarized probe pulse via the magneto-optical polar Kerr effect [15].

A schematics of the experimental setup is shown in Fig. 5. A mode-locked Ti:sapphire laser (Mai Tai, Spectra Physics) with a repetition rate $80 \mathrm{MHz}$ (i.e., a time separation between neighboring laser pulses of $t_{0}=12.5 \mathrm{~ns}$ ) is set to the wavelength $\lambda=815 \mathrm{~nm}$, close to the band gap of GaAs at low temperatures [30]. The generated approximately 100-fs laser pulses are split to pump and probe pulses, and their fluence is adjusted by neutral density filters (their intensity ratio at the sample is typically $10: 1$ ). A time delay between pump and probe pulses $\Delta t$ is controlled by a delay line from -0.5 to $3.5 \mathrm{~ns}$. The laser pulses are focused by a converging 10D lens on the sample to approximately $25-\mu \mathrm{m}$-sized overlapped spots. Unless explicitly mentioned otherwise,

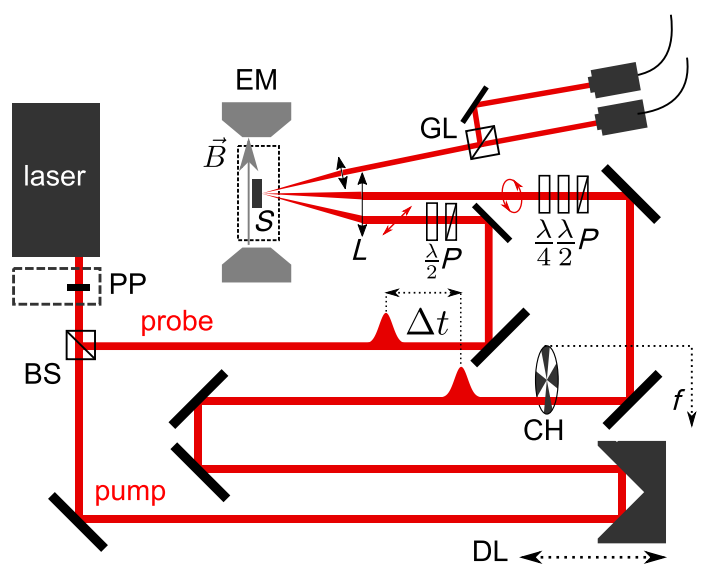

FIG. 5. A schematic depiction of the used pump-probe magneto-optical setup. Femtosecond laser pulses are split by a beam splitter (BS) to pump and probe pulses with a delay line (DL) controlled mutual time delay $(\Delta t)$. The required polarization state of laser pulses is set using a combination of polarizers $(P)$ and wave plates $(\lambda / 2$ and $\lambda / 4)$, and they are focused by a single lens $(L)$ on the sample $(S)$ placed in a cryostat. The reflected probe pulses are collimated by another lens, and their polarization rotation is measured by an optical bridge based on a Glan-Laser (GL) polarizer and a pair of silicon photodiode detectors. The difference electrical signal is processed by a lock-in amplifier at a modulation frequency $f$ of the optical chopper $(\mathrm{CH})$. External magnetic field $B$ is applied in the sample plane by an electromagnet (EM). Optionally, the pulse picker (PP) can be used to increase a time separation between neighboring laser pulses. the angles of incidence to the sample surface are $<1^{\circ}$ and approximately $7^{\circ}$ for the pump and probe beams, respectively. The sample is mounted in an optical cryostat at a temperature of $10 \mathrm{~K}$ and placed between the poles of an electromagnet that provides an in-plane magnetic field up to $B=500 \mathrm{mT}$ oriented in the plane of light incidence. The MO signal corresponding to the probe polarization rotation and the differential reflectivity are measured as a difference and sum signal in the optical bridge, respectively [31].

A pulse picker (PP) can be inserted to the setup at the position marked by "PP" in Fig. 5. A PP is essentially an electrically controlled acousto-optical modulator that transmit only certain pulses and block all the others. The PP in MO experiments is usually used in order to determine precisely the spin lifetime from MO time-resolved signals in systems where the spin-polarization decay time exceeds the time separation $t_{0}$ between the neighboring laser pulses. The dilution of laser pulses achieved by the PP is characterized by a so-called divider ratio $n$, which describes that the time separation between neighboring pulses is increased to $n t_{0}$. However, in the case of our experiments, we employ the PP in order to modulate the intensity of the pulse train instead of the selection of every $n$th pulse. This is possible thanks to the unideal efficiency of the pulse suppression by the PP. We verify experimentally that the diminished laser pulses have intensity suppressed at least 30 times, which is the detection limit given by the noise level in our measurement with a fast photodiode, with respect to that of the unattenuated ones. The diminished laser pulses create the spin-polarized population in the subsystem which is already saturated with respect to the laser fluence [see the inset in Fig. 4(a)]. This means that all states which are available in the confined system are already filled by the diminished excitation pulse.

The polar Kerr effect, which is the origin of the detected probe polarization rotation in our sample, changes a sign around the semiconductor band gap — see the inset in Fig. 1 in Ref. [32]. Consequently, if spectrally broad laser pulses are used, which is the case for our approximately 100-fslong laser pulses (with a spectral bandwidth of approximately $10 \mathrm{~nm}$ ), the measured MO signals can be positive, negative, or even close to zero depending on the mutual spectral position of the laser pulse and the MO spectrum. If more than one MO-active system with a distinct $\mathrm{MO}$ spectrum is present in the sample, the fine-tuning of the probe pulses can be used to enhance or suppress the sensitivity of the detected MO signal to the system of interest. This is the reason why the spins photoinjected to the bulk region outside the confined subsystem, which are rather short lived, do not significantly contribute to the MO signals shown in this paper, which were optimized with respect to the confined electron subsystem-see Figs. 1(c) and 4(a). More discussion about this can be found in Supplemental Material in Ref. [16]. 


\section{APPENDIX B: ESTIMATE OF DEGREE OF ELECTRON SPIN POLARIZATION FROM NUCLEAR POLARIZATION MEASUREMENTS}

The method elaborated in this Appendix makes use of approaches developed in Refs. [10,33-35].

In semiconductors whose nuclei carry a magnetic moment, like GaAs, the degree of spin polarization of electrons, $P_{e}$, can be transferred from electrons to nuclei via the hyperfine interaction. The evolution of the degree of polarization of nuclei $P_{n}$ in the laboratory time $t$ follows the exponential function [36-38]

$$
P_{n}(t) \propto P_{e}\left(1-e^{-t / T_{n}}\right),
$$

where $T_{n}$ is the characteristic time of the electron-nuclear hyperfine coupling, which is usually of the order of minutes [36-39] (i.e., much longer that the electron spin-relaxation time). Consistently with the main text, we use the standard definition of the degree of polarization [23], $P=\left(n_{+}-n_{-}\right) /$ $\left(n_{+}+n_{-}\right)$, with respect to the density $n_{+}$or $n_{-}$of spin carriers with a spin oriented parallel or antiparallel to the quantization axis (propagation vector of the circularly polarized excitation beam).

As the nuclei are getting polarized, they generate a nuclear magnetic field $\mathbf{B}_{n}=b_{n}\left\langle\mathbf{P}_{n}\right\rangle$ that adds to the external magnetic field $\mathbf{B}$. Here, $b_{n}=-5.3 \mathrm{~T}$ is the magnetic field that would be generated by fully spinpolarized nuclei, and $\mathbf{P}_{n}=P_{n} \hat{\mathbf{I}}$ is the vector of nuclear spin polarization oriented along the unit vector of the nuclear spin $\hat{\mathbf{I}}$. In our experiment, we use the optical generation of electrons with average spin polarization $P_{e}$ to polarize the nuclei. In saturation at $t \gg T_{n}, \mathbf{B}_{n}$ can be rewritten in terms of $P_{e}$ as $[33,34]$

$$
\mathbf{B}_{n}=f b_{n}^{*} \frac{\mathbf{B} \cdot \mathbf{P}_{e}}{B^{2}} \mathbf{B}
$$

where the vector $\mathbf{P}_{e}=P_{e} \hat{\mathbf{s}}$ is oriented along the unit vector of the electronic spin $\hat{\mathbf{s}}\left(P_{e}=1\right.$ for a fully spin-polarized electronic system), $b_{n}^{*}=-8.5 \mathrm{~T}$ is the effective magnetic field generated by a spin-polarized nuclei [40], and $f \leq 1$ is a phenomenological leakage factor [33] that takes into account possible processes of nuclear spin relaxation other than the hyperfine coupling with electrons. Although $f$ can be sample dependent, the value $f b_{n}^{*} \approx-0.85 \mathrm{~T}$ is usually reported and/or used in related calculations [33,37,41,42]. Equation (B2) holds for $B \gg 1 \mathrm{mT}$ when the nuclear dipole-dipole interactions are negligible. Consequently, if the angle between $\mathbf{B}$ and $\mathbf{P}_{e}$ is known, the value of $P_{e}$ can be inferred from the measured value of $B_{n}$.

In our experimental setup, the angles of incidence to the sample surface are $<1^{\circ}$ and approximately $7^{\circ}$ for the pump and probe beams, respectively (see Appendix A). However, the polarization of nuclei happens only if there is a nonzero
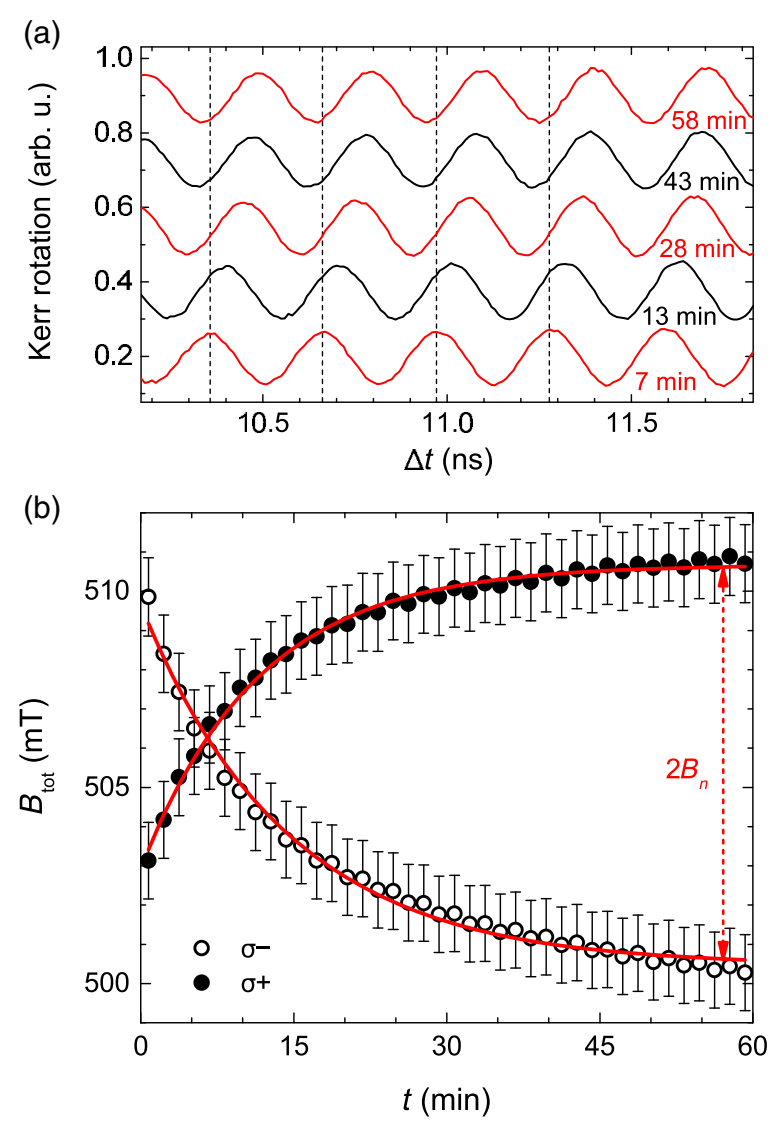

FIG. 6. (a) Time-resolved MO signal measured on the sample whose nuclei have been spin polarized by long-term exposure to light with the helicity $\sigma_{+}$. The curves and the corresponding labels indicate the laboratory time during which the sample is illuminated by light with the helicity $\sigma_{-}$; the curves are vertically shifted for clarity. The vertical lines emphasize the variation of the precession frequency due to the varying nuclear magnetic field. Data are measured for the time separation between neighboring pulses $n t_{0}=12.5 \mathrm{~ns}$, excitation fluence $14 \mu \mathrm{J} \mathrm{cm}^{-2}$, sample temperature $T=10 \mathrm{~K}$, and external magnetic field $B=500 \mathrm{mT}$. (b) The dependence of $B_{\mathrm{tot}}=B+B_{n}$ on the laboratory time for excitation by helicities $\sigma_{-}$(open symbols) and $\sigma_{+}$(closed symbols) measured on the sample whose nuclei have been spin polarized by long-term exposure to light with the opposite helicity. The curves are the fits by Eq. (B3). The vertical arrow depicts the saturated value of $2 B_{n}$.

component of the optically injected spins in the direction of the magnetic field, lying in the sample plane in our experimental configuration [see Eq. (B2)]. So, the angle between $\mathbf{B}$ (the sample plane) and $\mathbf{P}_{e}$ (the direction of propagation of the pump beam) has to be different from $90^{\circ}$ -i.e., the angle of incidence of the pump beam has to be nonzero for an efficient polarization of the nuclei. To achieve this, we exchange the role which the optical beams play in our setup (see Fig. 5). Consequently, the angle of incidence of the pump beam in this configuration is approximately $7^{\circ}$, and the range of accessible time delays is shifted to $\Delta t=9-13 \mathrm{~ns}$. 
Since the spin transfer from electrons to nuclei via the hyperfine channel is a rather slow process whose characteristic time $T_{n}$ is of the order of minutes or tens of minutes [36-39], the process of nuclei polarization can be measured directly in laboratory time. As an example, in Fig. 6(a) is shown a sequence of time-resolved MO signals measured for $\sigma_{-}$pump helicity at depicted times after a long-term sample exposure to light with the helicity $\sigma_{+}$. Clearly, we observe a gradual change of the precession frequency $\Omega=$ $g \mu_{B} B_{\text {tot }} / \hbar$ due to the increase of the total magnetic field $B_{\text {tot }}=B+B_{n}$ experienced by spin-polarized electrons. In Fig. 6(b), we show the values of $B_{\text {tot }}$ inferred from the fits of measured dynamics as a function of the laboratory time for both helicities of pump pulses. We observe that the nuclear polarization saturation occurs after $t \approx 60 \mathrm{~min}$. This time is roughly one order of magnitude longer than the values reported in the literature for $n$-doped GaAs (with $n \approx 2-5 \times 10^{16} \mathrm{~cm}^{-3}$ ) [37,39]. Considering that charging times longer than tens of minutes are reported in undoped GaAs [38], these data suggest a low density of spinpolarized electrons in the subsystem. This is in agreement with the corresponding electron concentration of the order of approximately $10^{15} \mathrm{~cm}^{-3}$ which is determined experimentally for this sample in Ref. [16].

Following Eq. (B1), the precise value of $B_{n}$ of the saturated nuclear polarization, which is depicted as a vertical arrow in Fig. 6(b), can be determined by fitting the deduced values of $B_{\text {tot }}$ for both helicities by

$$
B_{\mathrm{tot}}(t)=B_{\mathrm{tot}}^{ \pm} \pm C^{ \pm} e^{-t / T_{n}^{ \pm}} .
$$

Here, $B_{\text {tot }}^{ \pm}$are the saturation total magnetic fields for the excitation with $\sigma_{ \pm}, C^{ \pm}$are the corresponding amplitudes of the charging process, and $T_{n}^{ \pm}$are the characteristic times. We note that $\mathbf{B}_{n}$ is parallel or antiparallel to $\mathbf{B}$ with respect to the excitation by $\sigma_{+}$and $\sigma_{-}$. Considering the error bars, the fits give $B_{n}=\left(B_{\text {tot }}^{+}-B_{\text {tot }}^{-}\right) / 2=5.1 \pm 0.5 \mathrm{mT}$ and the average $T_{n}=(13 \pm 1) \mathrm{min}$. Finally, the degree of electron spin polarization $P_{e} \approx 18 \%$ is estimated using Eq. (B2). Here, we consider that the pump light refracts on the airGaAs interface and use $\mathbf{B} \cdot \mathbf{P}_{e}=B P_{e} \sin \alpha / n$, where $\alpha \approx 7^{\circ}$ is the angle of incidence of the pump light and $n=3.67$ the refractive index of GaAs at $\lambda=815 \mathrm{~nm}$.

[1] Claude Chappert, Albert Fert, and Frédéric Nguyen Van Dau, The emergence of spin electronics in data storage, Nat. Mater. 6, 813 (2007).

[2] J. Wunderlich, B. G. Park, A. C. Irvine, L. P. Zârbo, E. Rozkotová, P. Nemec, V. Novák, J. Sinova, and T. Jungwirth, Spin Hall effect transistor, Science 330, 1801 (2010).

[3] S. A. Wolf, D. D. Awschalom, R. A. Buhrman, J. M. Daughton, S. Von Molnar, M. L. Roukes, A. Yu Chtchelkanova, and D. M. Treger, Spintronics: A spin-based electronics vision for the future, Science 294, 1488 (2001).
[4] Supriyo Datta and Biswajit Das, Electronic analog of the electro-optic modulator, Appl. Phys. Lett. 56, 665 (1990).

[5] S. D. Sarma, Spintronics: A new class of device based on electron spin, rather than on charge, may yield the next generation of microelectronics, Am. Sci. 89, 516 (2001).

[6] M. B. A. Jalil, S. G. Tan, and T. Fujita, Spintronics in 2DEG systems, AAPPS Bull. 18, 9 (2008).

[7] J. Wunderlich, A. C. Irvine, J. Sinova, B. G. Park, L. P. Zârbo, X. L. Xu, B. Kaestner, V. Novák, and T. Jungwirth, Spin-injection Hall effect in a planar photovoltaic cell, Nat. Phys. 5, 675 (2009).

[8] L. Nádvorník, J. A. Haigh, K. Olejník, A. C. Irvine, V. Novák, T. Jungwirth, and J. Wunderlich, Efficient conversion of light to charge and spin in Hall-bar microdevices, Phys. Rev. B 91, 125205 (2015).

[9] L. Nádvorník, K. Olejník, P. Němec, V. Novák, T. Janda, J. Wunderlich, F. Trojánek, and T. Jungwirth, Enhancement of the spin Hall voltage in a reverse-biased planar $\mathrm{p}-\mathrm{n}$ junction, Phys. Rev. B 94, 075306 (2016).

[10] J. M. Kikkawa and D. D. Awschalom, Resonant Spin Amplification in n-Type GaAs, Phys. Rev. Lett. 80, 4313 (1998).

[11] R. I. Dzhioev, K. V. Kavokin, V. L. Korenev, M. V. Lazarev, B. Y. Meltser, M. N. Stepanova, B. P. Zakharchenya, D. Gammon, and D. S. Katzer, Low-temperature spin relaxation in n-type GaAs, Phys. Rev. B 66, 245204 (2002).

[12] D. Sprinzl, P. Horodyská, N. Tesařová, E. Rozkotová, E. Belas, R. Grill, P. Malý, and P. Němec, Influence of n -type doping on electron spin dephasing in CdTe, Phys. Rev. B 82, 153201 (2010).

[13] M. W. Wu, J. H. Jiang, and M. Q. Weng, Spin dynamics in semiconductors, Phys. Rep. 493, 61 (2010).

[14] V. M. Agranovich, F. Meier,, B. P. Zakharchenya, and A. A. Maradudin, Optical Orientation, Modern Problems in Condensed Matter Sciences Vol. 8 (Elsevier Science, Amsterdam, 1984).

[15] T. Korn, Time-resolved studies of electron and hole spin dynamics in modulation-doped GaAs/AlGaAs quantum wells, Phys. Rep. 494, 415 (2010).

[16] L. Nádvorník, P. Němec, T. Janda, K. Olejník, V. Novák, V. Skoromets, H. Němec, P. Kužel, F. Trojánek, T. Jungwirth, and J. Wunderlich, Long-range and high-speed electronic spin-transport at a GaAs/AlGaAs semiconductor interface, Sci. Rep. 6, 22901 (2016).

[17] Based on our calculations made in Supplemental Material in Ref. [16], we estimate the density of surface states $N_{s} \sim$ $10^{11}-10^{12} \mathrm{~cm}^{-2}$ and bulk impurity states $N_{b} \sim 10^{15} \mathrm{~cm}^{-3}$.

[18] The free nature of the confined electrons is also directly proved by our previous transport experiments in Ref. [16], where we demonstrate high-mobility and diffusion properties of these carriers.

[19] W. Zawadzki, P. Pfeffer, R. Bratschitsch, Z. Chen, S. T. Cundiff, B. N. Murdin, and C. R. Pidgeon, Temperature dependence of the electron spin g-factor in GaAs, Phys. Rev. B 78, 245203 (2008).

[20] P. Nemec, Y. Kerachian, H. M. van Driel, and Arthur L. Smirl, Spin-dependent electron many-body effects in GaAs, Phys. Rev. B 72, 245202 (2005).

[21] M. J. Snelling, G. P. Flinn, A. S. Plaut, R. T. Harley, A. C. Tropper, R. Eccleston, and C. C. Phillips, Magnetic g factor 
of electrons in GaAs/AlxGa1-xAs quantum wells, Phys. Rev. B 44, 11345 (1991).

[22] The rate equation for an energy level with a maximum density of available states $N$ leads to $n_{s}=(N) /$ $\left[1+N /\left(G \tau_{s}\right)\right]$, where $n_{s}$ is the density of spins in the subsystem and $G$ the generation rate. Since $n_{s}$ and $G$ are proportional to $A_{1}$ and $I$, respectively, we can use the model to fit the experimental dependence. However, as the corresponding proportionality constants are unknown, it is not possible to deduce any physically meaningful information from the fitting parameters.

[23] Igor Žutić, Jaroslav Fabian, and S. Das Sarma, Spintronics: Fundamentals and applications, Rev. Mod. Phys. 76, 323 (2004).

[24] P. Nahálková, P. Němec, D. Sprinzl, E. Belas, P. Horodyský, J. Franc, P. Hlídek, and P. Malý, Spin dynamics in bulk CdTe at room temperature, Mater. Sci. Eng. B 126, 143 (2006).

[25] R. D. R. Bhat, P. Nemec, Y. Kerachian, H. M. van Driel, J. E. Sipe, and Arthur L. Smirl, Two-photon spin injection in semiconductors, Phys. Rev. B 71, 035209 (2005).

[26] Y. S. Chen, S. Fält, W. Wegscheider, and G. Salis, Unidirectional spin-orbit interaction and spin-helix state in a (110)-oriented GaAs/(Al, Ga)As quantum well, Phys. Rev. B 90, 121304 (2014).

[27] R. Völkl, M. Schwemmer, M. Griesbeck, S. A. Tarasenko, D. Schuh, W. Wegscheider, C. Schüller, and T. Korn, Spin polarization, dephasing, and photoinduced spin diffusion in (110)-grown two-dimensional electron systems, Phys. Rev. B 89, 075424 (2014).

[28] I. Y. Gerlovin, Y. P. Efimov, Y. K. Dolgikh, S. A. Eliseev, V. V. Ovsyankin, V. V. Petrov, R. V. Cherbunin, I. V. Ignatiev, I. A. Yugova, L. V. Fokina, A. Greilich, D. R. Yakovlev, and M. Bayer, Electron-spin dephasing in GaAs/AlGaAs quantum wells with a gate-controlled electron density, Phys. Rev. B 75, 115330 (2007).

[29] A. V. Kimel, V. V. Pavlov, R. V. Pisarev, V. N. Gridnev, F. Bentivegna, and Th Rasing, Ultrafast dynamics of the photo-induced magneto-optical Kerr effect in CdTe at room temperature, Phys. Rev. B 62, R10610 (2000).

[30] M. Surynek et al. (to be published).

[31] E. Rozkotová, P. Němec, N. Tesařová, P. Malý, V. Novák, K. Olejník, M. Cukr, and T. Jungwirth, Coherent control of magnetization precession in ferromagnetic semiconductor (Ga,Mn)As, Appl. Phys. Lett. 93, 232505 (2008).
[32] S. A. Crooker and D. L. Smith, Imaging Spin Flows in Semiconductors Subject to Electric, Magnetic, and Strain Fields, Phys. Rev. Lett. 94, 236601 (2005).

[33] D. Paget, G. Lampel, B. Sapoval, and V. I. Safarov, Low field electron-nuclear spin coupling in gallium arsenide under optical pumping conditions, Phys. Rev. B 15, 5780 (1977).

[34] G. Salis, A. Fuhrer, and S. F. Alvarado, Signatures of dynamically polarized nuclear spins in all-electrical lateral spin transport devices, Phys. Rev. B 80, 115332 (2009).

[35] M. I. Dyakonov and V. I. Perel, Optical orientation in a system of electrons and lattice nuclei in semiconductors. Theory, Sov. Phys. JETP 38, 177 (1974).

[36] A. Greilich, A. Shabaev, D. R. Yakovlev, Al. L. Efros, I. A. Yugova, D. Reuter, A. D. Wieck, and M. Bayer, Nucleiinduced frequency focusing of electron spin coherence, Science 317, 1896 (2007).

[37] J. Shiogai, M. Ciorga, M. Utz, D. Schuh, T. Arakawa, M. Kohda, K. Kobayashi, T. Ono, W. Wegscheider, D. Weiss, and J. Nitta, Dynamic nuclear spin polarization in an all-semiconductor spin injection device with (Ga,Mn)As/ n-GaAs spin Esaki diode, Appl. Phys. Lett. 101, 212402 (2012).

[38] K. Hashimoto, K. Muraki, T. Saku, and Y. Hirayama, Electrically Controlled Nuclear Spin Polarization and Relaxation by Quantum-Hall States, Phys. Rev. Lett. 88, 176601 (2002).

[39] Daniel Paget, Optical detection of NMR in high-purity GaAs: Direct study of the relaxation of nuclei close to shallow donors, Phys. Rev. B 25, 4444 (1982).

[40] The value of $b_{n}^{*}=-8.5 \mathrm{~T}$ (used in the formulation of $B_{n}$ in terms of $P_{e}$ ) differs from $b_{n}=-5.3 \mathrm{~T}$ (the formulation in terms of $P_{n}$ ). This is due to a different projection of spins of electrons $(j=1 / 2)$ and nuclei $(j=1 / 2,3 / 2)$, which results in that $P_{n}$ is not a linear function of $P_{e}$ (see Fig. 1 in Ref. [35]). Consequently, the value of $b_{n}^{*}$ varies from -8.5 to $-5.3 \mathrm{~T}$ depending on the value of $P_{e}$.

[41] K. D. Christie, C. C. Geppert, S. J. Patel, Q. O. Hu, C. J. Palmstrøm, and P. A. Crowell, Knight shift and nuclear spin relaxation in Fe/n-GaAs heterostructures, Phys. Rev. B 92, 155204 (2015).

[42] G. Sallen, S. Kunz, T. Amand, L. Bouet, T. Kuroda, T. Mano, D. Paget, O. Krebs, X. Marie, K. Sakoda, and B. Urbaszek, Nuclear magnetization in gallium arsenide quantum dots at zero magnetic field, Nat. Commun. 5, 3268 (2014). 